\title{
Equilibrium, observability and controllability in selection-mutation models
}

\author{
I. López ${ }^{a}$ M. Gámez ${ }^{a}$ Z. Varga ${ }^{\text {b }}$ \\ ${ }^{a}$ Department of Statistics and Applied Mathematics, University of Almeria, Spain \\ b Institute of Mathematics and Informatics, Szent István University, Gödöllö, \\ Hungary.
}

\begin{abstract}
In this paper we shortly discuss the problem of the equilibrium in the well-known Fisher type selection-model, also providing a formula for particular three-allele models. The considered continuous-time dynamics is a known extension of the classical model of natural selection given by Fisher. We also extend the existing investigation of the observability of Fisher's model to the case when another evolutionary factor, mutation is also present. Moreover, we prove a result of technical character, which makes it possible to apply the methodology of nonlinear systems with invariant manifold, to models of artificial selection. For an illustration, a class of three-allele systems is presented in which the controllability into equilibrium is guaranteed without any condition on the biological parameters.
\end{abstract}

Key words: selection-mutation dynamics, observability of nonlinear systems, controllability of nonlinear systems

\section{Introduction}

Alleles can mutation during DNA replication in meiosis. Mutations that occur are a source of genetic variation, despite the action of selection. Mutant alleles are maintained by neutral drift or a lack of direct selection pressure.

\footnotetext{
Email addresses: milopez@ual.es corresponding author (I. López), mgamez@ual.es (M. Gámez), Varga.Zoltan@gek.szie.hu (Z. Varga).
} 
Therefore, in a one-locus diploid model, we consider the simultaneous action of selection and mutation on the dynamics, where the effects of the latter, being small in general, change the allelic frequencies in a linear way. We study the well-known Fisher type selection-mutation model from the view point of mathematical systems theory. This model is an extension of the classical continuous-time model of natural selection given by Fisher $(1930,1958)$, and studied by many authors. In the following sections, we shall also discuss the model, according to the absence or presence of mutation.

Fundamental results concerning the basic selection-mutation model can be found in Crow and Kimura (1970); Moran (1976, 1977); Hadeler (1981). A general survey of the most recent advances in the study of the mathematical properties of these models is Bürger (1998). Concerning the Fisher type dynamics, in Section 2 we shall discuss the problem of the existence and simple calculation of a polymorphic equilibrium (i.e., an equilibrium in the interior of the standard simplex). Recalling a sufficient condition for the existence of a common equilibrium for both the pure selection and selection-mutation models, we present a case where when this condition doesn't verify, and the equilibria of the two models are different.

We also raise the problem of whether or not we can recover the whole genetic process from the observation of certain phenotypic characteristics of the population undergoing a selection-mutation process. (We notice that, in static situation, in Garay and Garay (1998) for the one-locus case biological conditions were given to guarantee the genotypic state-phenotypic state correspondence to be one to one). We shall also discuss the problem whether we can control the population into equilibrium changing certain parameters of the population. These two problems are related to the concepts of observability and controllability well-known in mathematical systems theory. This discipline had been developed by the 1960s to deal with a variety of applications in engineering and industry, see Zadeh and Desoer (1963) and Kalman et al. (1969).

Mathematical systems theory has become quite familiar to system engineers, observability and controllability analysis of dynamic models in biology is relatively new. A sufficient condition for local controllability has been proved in Varga (1989) where this condition was applied to the selection model of Fisher. Later, this mathematical result turned out to be a useful tool in the system- 
theoretical investigation of evolutionary game dynamics (Kósa and Varga, 1996; Gámez et al., 2003; López et al., 2003), and reaction kinetics models (Farkas, 1998a). Similar results for observability have also been obtained in Varga (1992), and applied to frequency-dependent evolutionary models (López et al., 2002) and to other fields, as well (see Farkas 1998b). In Section 3 results on observability in the selection-mutation model are presented. Mathematical systems theory has become quite familiar to system engineers, observability and controllability analysis of dynamic models in biology is relatively new. For applications to ecological monitoring see e. g. Varga et al. (2003).

In Scarelli and Varga (2002) it is supposed that certain mutation rates can be changed and considered as time-dependent control variables, and a method is offered which provides sufficient conditions for the local controllability of selection-mutation process to a polymorphic equilibrium. (For the methods of "self-adaptation", closely related to the control of mutation rates, see Fogel 2000). For another way to control the selection-mutation process, we will propose to consider time-dependent fitness parameters as control functions. A model of this type can be interpreted as the dynamic description of artificial selection.

\section{Equilibrium in the selection-mutation model}

In this section, we shall deal with condition which guarantee the existence of a polymorphic equilibrium in the selection-mutation model and we shall also check whether this equilibrium is different for the pure selection model of Fisher, when there is no mutation.

First, we recall the Fisher type selection-mutation model. We consider a diploid population with random mating and with alleles $A_{1}, A_{2}, \ldots, A_{n}$ at an autosomal locus. Assume that the individuals undergo a selection described in terms of a fitness matrix $W \in \mathbb{R}^{n \times n}$ with non-negative entries where, for each $i, j \in\{1, \ldots, n\}, w_{i j}$ is the fitness value of an $A_{i} A_{j}$ zygote (by the Mendelian symmetry, $W$ is symmetric). Then according to Fisher's classical model of natural selection, for the frequency $x_{i}$ of allele $A_{i}$ we have the following system of differential equations

$$
\dot{x}_{i}=x_{i}\left[(W x)_{i}-\bar{w}(x)\right] \quad i \in\{1,2, \ldots, n\},
$$


where $(W x)_{i}=\sum_{j=1}^{n} w_{i j} x_{j}$ is the potencial (marginal) fitness of allele $A_{i}$, while $\bar{w}: \Delta_{n} \rightarrow \mathbb{R}$ with $\bar{w}(x)=\sum_{i, j=1}^{n} w_{i j} x_{i} x_{j}$ is the mean fitness of the population.

Now, for each $i, j \in\{1,2, \ldots, n\}, i \neq j$, let the non-negative number $m_{i j}$ be the rate of mutation from allele $A_{j}$ to allele $A_{i}$, and define,

$$
m_{i i}:=-\sum_{j \neq i} m_{j i}
$$

Then, in terms of the fitness matrix $W$ and the mutation matrix $M:=$ $\left(m_{i j}\right)_{n \times n}$, the selection-mutation process is described by the following system of differential equations

$$
\dot{x}_{i}=x_{i}\left[(W x)_{i}-\bar{w}(x)\right]+(M x)_{i} \quad i \in\{1,2, \ldots, n\}
$$

for the vector $x$ of allele frequencies of the population as function of time.

Concerning the existence and stability of a polymorphic equilibrium, under the condition of a special structure of the mutation matrix (when the mutation rates depend only on the target allele), extending a result of Hadeler (1981), Hofbauer (1985) proved the following: if the pure selection (with $M \equiv 0$ ) model has an asymptotically stable polymorphic equilibrium, then system (2) has a unique equilibrium which is globally asymptotically stable. Moreover, by a theorem of Akin (1979), the given restriction in the mutation matrix can not be dropped.

The problem of the existence and stability of equilibria in the continuous-time selection-mutation model has been studied in Varga and Zubiri (1993). Using Brower's fix point theorem, it was proved that if the selection is weak (the variation of fitness is not too large) and the mutation rates are sufficiently small, then there exists an equilibrium in the standard simplex $\Delta_{n}$, moreover the effective calculation of the equilibrium is also possible under certain conditions, see also Scarelli and Varga (2002):

Theorem 2.1. Suppose that:

(i) $W$ is invertible;

(ii) For the vector $\mathbf{1}:=(1, \ldots, 1) \in \mathbb{R}^{n}$ we have $<W^{-1} \mathbf{1}, \mathbf{1}>\neq 0$;

(iii)

$$
x_{i}^{*}:=\left(\frac{W^{-1} \mathbf{1}}{<W^{-1} \mathbf{1}, \mathbf{1}>}\right)_{i}>0 \quad i \in\{1, \ldots, n\}
$$

(iv) $W^{-1} \mathbf{1} \in \operatorname{Ker} M$. 
Then $x^{*}=\left(x_{1}^{*}, \ldots, x_{n}^{*}\right)$ is a polymorphic equilibrium for dynamics (2).

Remark 1. It is known (see Varga (1992)) that if we suppose

$$
\begin{gathered}
\operatorname{det} W \neq 0, \quad<W^{-1} \mathbf{1}, \mathbf{1}>\neq 0 \\
x^{*}=\frac{W^{-1} \mathbf{1}}{<W^{-1} \mathbf{1}, \mathbf{1}>} \in \text { int } \mathbb{R}_{+}^{3},
\end{gathered}
$$

then $x^{*}$ is the unique polymorphic equilibrium of pure selection model ( $M=$ 0 ). Therefore, the condition (iv) of Theorem 2.1 is a simple sufficient condition to guarantee that the polymorphic equilibrium of pure selection model is at the same time an equilibrium of the selection-mutation model. In the following example, for a class of models we shall see that if condition (iv) is not satisfied then this coincidence may not occur.

Example 2.2. In a two-allele model, suppose that $W$ is a symmetric and invertible matrix and there is no mutation from allele $A_{2}$ to allele $A_{1}$. Now we shall see that if the conditions

$$
\begin{aligned}
& w_{12}-w_{11}>0 \\
& w_{12}-w_{22}>m_{21}>0,
\end{aligned}
$$

are fulfilled, then the equilibrium of system (2) is different from that of the case of pure selection $(M=0)$.

Firstly, since $m_{12}=m_{22}=0$ by hypothesis, and by definition we have $m_{11}=$ $-m_{21}$, the mutation matrix has the form

$$
M=\left(\begin{array}{rr}
-a & 0 \\
a & 0
\end{array}\right) \quad \text { with } \quad a:=m_{21} \text {. }
$$

By $x_{2}=1-x_{1}$, instead of the system (2), it is sufficient to consider the equation for $x_{1}$, which takes the form

$$
\dot{x}_{1}=x_{1}^{3}\left(2 w_{12}-w_{11}-w_{22}\right)+x_{1}^{2}\left(w_{11}-3 w_{12}+2 w_{22}\right)+x_{1}\left(w_{12}-w_{22}-a\right) .
$$

Defining $K:=2 w_{12}-w_{11}-w_{22} ; p:=w_{12}-w_{22}$, by hypotheses (4) and (5) we have $K, p>0$, and it is easy to verify that $(K+p)^{2}-4 K(p-a)>0$. A simple calculation shows that the unique non-trivial equilibrium of (6) can be

$$
x_{1}^{*}=\frac{K+p-\sqrt{(K+p)^{2}-4 K(p-a)}}{2 K} .
$$


Since $p-a>0$, from (7) we get $x_{1}^{*}>0$.

Moreover, obviously $K>p$ and $(K+p)^{2}-4 K(p-a)=(K-p)^{2}+4 K a$. Therefore,

$$
x_{1}^{*}=\frac{K+p-\sqrt{(K-p)^{2}+4 K a}}{2 K}<\frac{(K+p)-(K-p)}{2 K}=1,
$$

is a non-trivial equilibrium.

If we calculate the non-trivial equilibrium of the pure selection model, we obtain

$$
y_{1}^{*}=\left(\frac{W^{-1} \mathbf{1}}{<W^{-1} \mathbf{1}, \mathbf{1}>}\right)_{1}=\frac{w_{22}-w_{12}}{w_{11}-2 w_{12}+w_{22}}=\frac{p}{K}>0
$$

which is obviously different from $x_{1}^{*}$, if $a>0$. Of course, from (7), with $a=0$ we recover $x_{1}^{*}=y_{1}^{*}$.

The conditions (4) and (5) imply a situation of heterosis: the heterozygotes are fitter than the homozygotes. It can be proved that $y_{1}^{*}$ represent an asymptotically stable equilibrium in the pure selection model. (For a similar case see Varga and Zubiri, 1993).

Now we consider the selection-mutation model (2) for $n=3$ with selection and mutation matrices

$$
W=\left(\begin{array}{lll}
w_{11} & w_{11} & w_{13} \\
w_{11} & w_{22} & w_{13} \\
w_{13} & w_{13} & w_{33}
\end{array}\right) \text { and } \quad M=\left(\begin{array}{rrr}
-a & 0 & 0 \\
0 & -b & 0 \\
a & b & 0
\end{array}\right), \quad a, b>0 .
$$

In the following theorem we give an explicit formula to calculate a polymorphic equilibrium of the selection-mutation process, under certain conditions: condition ( $i$ ) is a kind of fitness additivity, (ii) says to what extent a heterozygote must be fitter than a homozygote, while (iii) is a generic condition posed on the model parameters.

Theorem 2.3. If the following conditions are verified:

(i) $w_{11}+w_{33}=2 w_{13}$,

(ii) $w_{13}-w_{33}>a>b$,

(iii) $\frac{\left(w_{13}-w_{33}-a\right)\left(a-b+w_{22}-w_{11}\right)}{b(b-a)}>1$. 
Then $x^{*}=\left(x_{1}^{*}, x_{2}^{*}, x_{3}^{*}\right)$ with

$$
x_{2}^{*}=\frac{b-a}{w_{22}-w_{11}}, \quad x_{3}^{*}=\frac{(b-a) x_{2}^{*}+a}{w_{13}-w_{33}}, \quad x_{1}^{*}=1-x_{2}^{*}-x_{3}^{*}
$$

is a polymorphic equilibrium of system (2).

Proof. Since $x_{3}=1-x_{1}-x_{2}$, we shall consider only the first two equations of system (2), which by hypothesis $(i)$ takes the form

$$
\begin{aligned}
\dot{x}_{1}= & x_{1}\left[\left(-w_{13}+w_{33}\right) x_{1}+\left(-w_{13}+w_{33}\right) x_{2}\right. \\
& \left.+\left(-w_{22}+2 w_{13}-w_{33}\right) x_{2}^{2}+w_{13}-w_{33}-a\right] \\
\dot{x}_{2}= & x_{2}\left[\left(-w_{13}+w_{33}\right) x_{1}+\left(w_{22}-3 w_{13}+2 w_{33}\right) x_{2}\right. \\
& \left.+\left(-w_{22}+2 w_{13}-w_{33}\right) x_{2}^{2}+w_{13}-w_{33}-b\right]
\end{aligned}
$$

Searching a polymorphic equilibrium we conclude that the unique equilibrium with $x_{1} \neq 0$ and $x_{2} \neq 0$ can be the vector $x^{*}$ given in (9). Now we prove that under conditions $(i)-(i i i) x^{*}$ is a polymorphic equilibrium.

By hypothesis we have $b-a<0$, which with (ii) and (iii) imply that $w_{22}-$ $w_{11}<0$, and therefore $x_{2}^{*}>0$.

By hypothesis $(i i)$ we have that $w_{13}-w_{33}>0$. From (ii), (iii) and $b-a<0$ we obtain $a-b+w_{22}-w_{11}<0$, and therefore $x_{2}^{*}<1$, which with $a, b, x_{2}^{*}>0$ imply that

$$
(b-a) x_{2}^{*}+a=b x_{2}^{*}+a-a x_{2}^{*}>0 .
$$

Substituting the value of $x_{2}^{*}$ into $x_{3}^{*}$, we get

$$
x_{2}^{*}+x_{3}^{*}=\frac{(b-a)\left(w_{13}-w_{33}\right)+(b-a)^{2}+a\left(-2 w_{13}+w_{22}+w_{33}\right)}{\left(-2 w_{13}+w_{22}+w_{33}\right)\left(w_{13}-w_{33}\right)}
$$

Since by hypotheses $(i i)$ and $(i i i)$ we have

$$
w_{13}-w_{33}>0 \quad \text { and } \quad-2 w_{13}+w_{22}+w_{33}<0
$$

it is easy to see that all we have to prove is that

$$
(b-a)\left(w_{13}-w_{33}+b-a\right)+\left(-2 w_{13}+w_{22}+w_{33}\right)\left(a-w_{13}+w_{33}\right)>0,
$$


which is valied because

$$
b(b-a)+\left(w_{13}-w_{33}-a\right)\left(b-a+2 w_{13}-w_{22}-w_{33}\right)>0
$$

by hypothesis $($ iii) .

Remark 2. For a biological interpretation of Theorem 2.3 let us consider the case when the difference $a-b$ is small enough and the genotype $A_{1} A_{1}$ is fitter than $A_{2} A_{2}$. Then an easy calculation shows that the mathematical condition (iii) is satisfied. Therefore in this case we have the polymorphic equilibrium of (2).

Example 2.4. Consider the selection-mutation model (2) for $n=3$, with selection and mutation matrices

$$
W=\left(\begin{array}{lll}
3 & 3 & 2 \\
3 & 1 & 2 \\
2 & 2 & 1
\end{array}\right) \quad \text { and } \quad M=\left(\begin{array}{rrr}
-0.2 & 0 & 0 \\
0 & -0.1 & 0 \\
0.2 & 0.1 & 0
\end{array}\right)
$$

We check that the hypotheses of Theorem 2.3 hold. Indeed, clearly $a>b$, and

(i) $w_{11}+w_{33}=4=2 w_{13}$

(ii) $w_{13}-w_{33}=1>0.2$

(iii) $\frac{\left(w_{13}-w_{33}-a\right)\left(a-b+w_{22}-w_{11}\right)}{b(b-a)}=152>1$.

Therefore, applying Theorem 2.3, from formula (9) we get the polymorphic equilibrium

$$
x^{*}=(0.745,0.05,0.205) \text {. }
$$

\section{Observability in the selection-mutation model}

In this section we provide sufficient conditions for a selection-mutation model with a given allelic dominance structure to be locally observable. To this end, we consider model (2), and in order to simplify the calculations we put $n=3$. Moreover, in order to illustrate our reasoning, we consider an observation corresponding to a concrete dominance structure well-known in population genetics. 
Suppose that the dominance relations between three alleles $A_{1}, A_{2}$ and $A_{3}$ at an autosomal locus are the following: $A_{1}$ and $A_{2}$ are dominant over to $A_{3} ; A_{1}$ and $A_{2}$ are co-dominant. In other words, the genotypes $A_{1} A_{1}$ and $A_{1} A_{3}$ have the same phenotype $\overline{A_{1}} ; A_{2} A_{2}$ and $A_{2} A_{3}$ have the same phenotype $\overline{A_{2}} ; A_{1} A_{2}$ and $A_{3} A_{3}$ have the phenotypes $\overline{A_{1} A_{2}}$ and $\overline{A_{3}}$, respectively. A wellknown example for this dominance structure is the system of blood groups $A B O$. Assume that the frequencies of alleles $A_{1}, A_{2}$ and $A_{3}$ are $x_{1}, x_{2}$ and $x_{3}$, respectively (with $x=\left(x_{1}, x_{2}, x_{3}\right) \in \Delta_{3}$ ). Then, according to the HardyWeinberg law the frequencies of homozygote genotypes $A_{j} A_{j}$ are $x_{j}^{2}$ and that of the heterozygote genotypes $A_{i} A_{j}($ with $i \neq j)$ are $2 x_{i} x_{j}$.

Therefore, for any allele frequency vector $x \in \Delta_{3}$, the corresponding phenotype frequencies are the following:

$$
\begin{aligned}
& \overline{A_{1}}: x_{1}^{2}+2 x_{1} x_{3}, \\
& \overline{A_{2}}: x_{2}^{2}+2 x_{2} x_{3}, \\
& \overline{A_{1} A_{2}}: 2 x_{1} x_{2}, \\
& \overline{A_{3}}: x_{3}^{2} .
\end{aligned}
$$

Given the selection-mutation dynamics (2) and its polymorphic equilibrium $x^{*}=\left(x_{1}^{*}, x_{2}^{*}, x_{3}^{*}\right) \in \stackrel{\circ}{\Delta}_{3}$, we consider the observation of phenotype $\overline{A_{1} A_{2}}$, that is, for technical reason the difference between the actual frequency of this phenotype and its value at the equilibrium is observed. To this end, we introduce the following function

$$
h: \mathbb{R}^{3} \rightarrow \mathbb{R}, \quad h(x):=2 x_{1} x_{2}-2 x_{1}^{*} x_{2}^{*}
$$

In this way the observation system is

$$
\left\{\begin{array}{l}
\dot{x}_{i}=x_{i}\left[(W x)_{i}-\bar{w}(x)\right]+(M x)_{i} \quad i=\{1,2,3\} \\
y=2 x_{1} x_{2}-2 x_{1}^{*} x_{2}^{*}
\end{array}\right.
$$

For a consistent selection-mutation model we have the invariance of the standard simplex for dynamics (2) (see Hofbauer and Sigmund 1988, 1998). For the study of observability we shall also need the invariance of the interior of 
the simplex for this dynamics, which is a direct consequence of Lemma A1 of Appendix.

Definition 3.1. Given $k, n \in \mathbb{N}, k<n$, a set $N \subset \mathbb{R}^{n}$ is a regular $k$ dimensional sub-manifold of $\mathbb{R}^{n}$ if there exist an open set $G \subset \mathbb{R}^{n}$ and a continuously differentiable function $\phi: G \rightarrow \mathbb{R}^{n-k}$ such that, for the range of the derivative $\phi^{\prime}(x)$ we have $R_{\phi^{\prime}(x)}=\mathbb{R}^{n-k}(x \in G), 0 \in R_{G}$ and $N=\phi^{-1}(0)$.

For a continuously differentiable function $f: \mathbb{R}^{n} \rightarrow \mathbb{R}^{n}$, consider the system of differential equations

$$
\dot{x}=f \circ x,
$$

and suppose that $x^{*} \in N$ is an equilibrium for dynamics (11): $f\left(x^{*}\right)=0$. Given $m \in \mathbb{N}$, let $h: \mathbb{R}^{n} \rightarrow \mathbb{R}^{m}$ be a continuously differentiable function such that $h\left(x^{*}\right)=0$, then we define an observation system as

$$
\left\{\begin{array}{l}
\dot{x}=f \circ x \\
y=h \circ x .
\end{array}\right.
$$

$y$ is interpreted as an observed function for system (11).

Definition 3.2. For a given interval $[0, T]$, the submanifold $N$ is said to be locally positively invariant for (11) at $x^{*}$, if there exists $\delta>0$ such that for each solution $x$ of (11) with $x(0) \in N,\left|x(0)-x^{*}\right|<\delta$ we have $x(t) \in N(t \in[0, T])$. Definition 3.3. Suppose that $N$ is a regular $k$-dimensional, locally positively invariant sub-manifold for (11) at $x^{*}$. We shall say that the observation system (12) is locally observable in $N$ at point $x^{*}$ if there exists $0<\epsilon<\delta$ with the following property:

$$
\begin{aligned}
\text { If } z^{i} \in M, \| & z^{i}-x^{*} \|<\epsilon \quad(i \in\{1,2\}), \\
\dot{x}^{i}(t) & =f\left(x^{i}(t)\right) \quad(t \in[0, T]) \\
x^{i}(0) & =z^{i} \quad(i \in\{1,2\}) \\
h\left(x^{1}(t)\right) & =h\left(x^{2}(t)\right) \quad(t \in[0, T]),
\end{aligned}
$$

then $z^{1}=z^{2}$ (consequently $\left.x^{1}(t)=x^{2}(t) \quad(t \in[0, T])\right)$.

The local observability in $N$ at $x^{*}$ means that if, instead of the solution $x$ (the state of the considered object in function of time), we observe a transformation $y$ of it, then out of this observed function we can recover the solution in a unique way, provided the solution in question starts from a point of $N$ near the equilibrium $x^{*}$. 
Theorem 3.4. (Varga, 1992) Let $N \subset \mathbb{R}^{n}$ be a regular sub-manifold which is locally positively invariant at $x^{*}$. If

$$
T_{*} \cap \operatorname{Ker} Q=\{0\}
$$

then the system

$$
\left\{\begin{array}{l}
\dot{x}=f \circ x \\
y=h \circ x
\end{array}\right.
$$

is locally observable at the equilibrium $x^{*}$, where $T_{*}$ is the tangent space of $N$ at the point $x^{*}$ and $Q=\left(C|C L| \ldots \mid C L^{n-1}\right)^{\mathrm{T}}$ with $L:=f^{\prime}\left(x^{*}\right)$ and $C:=h^{\prime}\left(x^{*}\right)$.

Now we illustrate the application of this theorem to selection-mutation systems in the 3 -allele case (10).

Theorem 3.5. Suppose that for system (10), the following conditions hold.

$$
\begin{aligned}
x_{1}^{*} & =x_{2}^{*}, \\
w_{11} & =w_{22}, \quad w_{23} \geq w_{13}, \\
m_{32} & >m_{31} .
\end{aligned}
$$

Then system (10) is locally observable in $\stackrel{\circ}{\Delta}_{3}$ at the equilibrium $x^{*}$.

Proof. In order to apply Theorem 3.4, we consider an arbitrary $z \in T_{*} \cap \operatorname{Ker} Q$ and see that $z=0$, which guarantees that the considered system is locally observable.

First, if we calculate the matrix $C=h^{\prime}\left(x^{*}\right)$, we get

$$
C=2\left(x_{2}^{*}, x_{1}^{*}, 0\right)
$$

Since $z \in \operatorname{Ker} Q$, we have $C z=2\left(x_{2}^{*} z_{1}+x_{1}^{*} z_{2}\right)=0$. Hence by (14) and $x^{*} \in \stackrel{\circ}{\Delta}_{3}$ we obtain $z_{2}=-z_{1}$ and therefore $z_{3}=0$ because $z \in T_{*}$, that is, $z_{1}+z_{2}+z_{3}=0$.

Now, for the right-hand side $f$ we calculate matrix $L=\left(l_{i j}\right)_{3 \times 3}$, defined as $L=\frac{\partial f}{\partial x}\left(x^{*}\right)$ :

$$
l_{i j}=x_{i}^{*}\left[w_{i j}-2\left(W x^{*}\right)_{j}\right]+m_{i j} .
$$

Hence, for the coordinates of vector $[L z]_{3 \times 1}$ we obtain

$$
(L z)_{i}=\sum_{j} l_{i j} z_{j}=\sum_{j}\left(x_{i}^{*}\left[w_{i j}-2\left(W x^{*}\right)_{j}\right]+m_{i j}\right) z_{j} \quad i \in\{1,2,3\} .
$$


Therefore, by hypothesis (14), we get

$$
\begin{aligned}
C L z= & 2\left\{x_{1}^{*} \sum_{j}\left(x_{1}^{*}\left[w_{1 j}-2\left(W x^{*}\right)_{j}\right]+m_{1 j}\right) z_{j}\right. \\
& \left.+x_{1}^{*} \sum_{j}\left(x_{1}^{*}\left[w_{2 j}-2\left(W x^{*}\right)_{j}\right]+m_{2 j}\right) z_{j}\right\} \\
= & 2\left(x_{1}^{*}\right)^{2}\left\{\sum_{j}\left(w_{1 j}+w_{2 j}-4\left(W x^{*}\right)_{j}+\frac{m_{1 j}+m_{2 j}}{x_{1}^{*}}\right) z_{j}\right\} .
\end{aligned}
$$

Now, $z_{2}=-z_{1}$ and $z_{3}=0$ imply

$$
\begin{aligned}
C L z & =2\left(x_{1}^{*}\right)^{2} z_{1}\left(w_{11}-w_{22}+4\left[\left(W x^{*}\right)_{2}-\left(W x^{*}\right)_{1}\right]+\frac{m_{11}+m_{21}-m_{12}-m_{22}}{x_{1}^{*}}\right) \\
& =2\left(x_{1}^{*}\right)^{2} z_{1}\left\{w_{11}-w_{22}+4\left[\left(w_{22}-w_{11}\right) x_{1}^{*}+\left(w_{23}-w_{13}\right) x_{3}^{*}\right]+\frac{m_{11}+m_{21}-m_{12}-m_{22}}{x_{1}^{*}}\right\} .
\end{aligned}
$$

Hence by (15) and $z \in \operatorname{Ker} Q$ we also have $C L z=0$, that is,

$$
C L z=2\left(x_{1}^{*}\right)^{2} z_{1}\left[4\left(w_{23}-w_{13}\right) x_{3}^{*}+\frac{m_{11}+m_{21}-m_{12}-m_{22}}{x_{1}^{*}}\right]=0
$$

Furthermore, by definition

$$
m_{11}=-m_{21}-m_{31} \quad \text { and } \quad m_{22}=-m_{12}-m_{32}
$$

which together with condition (16) imply

$$
m_{11}+m_{21}-m_{12}-m_{22}=m_{32}-m_{31}>0 .
$$

Hence by $x^{*} \in \stackrel{\circ}{\Delta}_{3},(18)$ and hypothesis $w_{23} \geq w_{13}$ we obtain that

$$
4\left(w_{23}-w_{13}\right) x_{3}^{*}+\frac{m_{11}+m_{21}-m_{12}-m_{22}}{x_{1}^{*}}>0
$$

which together with (17) imply that $z_{1}=0$, and the proof is complete. 
Corollary 3.6. Given system (10), with mutation matrix

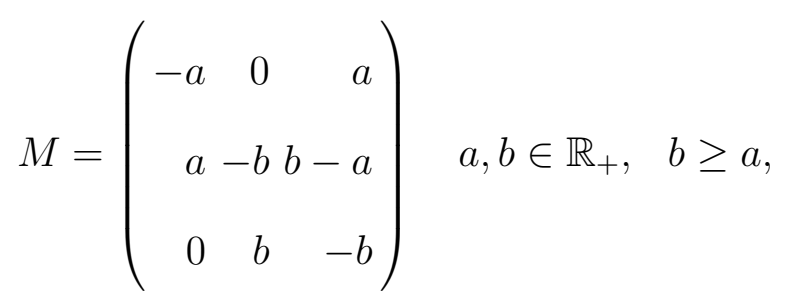

suppose that

$$
x_{1}^{*}=x_{2}^{*}, \quad w_{11}=w_{22}, \quad w_{23} \geq w_{13} .
$$

Then system (10) is locally observable in $\stackrel{\circ}{\Delta}_{3}$ at the equilibrium $x^{*}$.

Example 3.7. In Figure 1 we illustrate the observability of system (10) for the particular case when the fitness matrix is given by $w_{i i}=1(i=1,2,3)$ and $w_{i j}=0.5 \quad i \neq j \quad(i, j \in\{1,2,3\})$, and for the mutation matrix (19) we have $a=0.5$ and $b=1$, as obtained in Corollary 3.6.

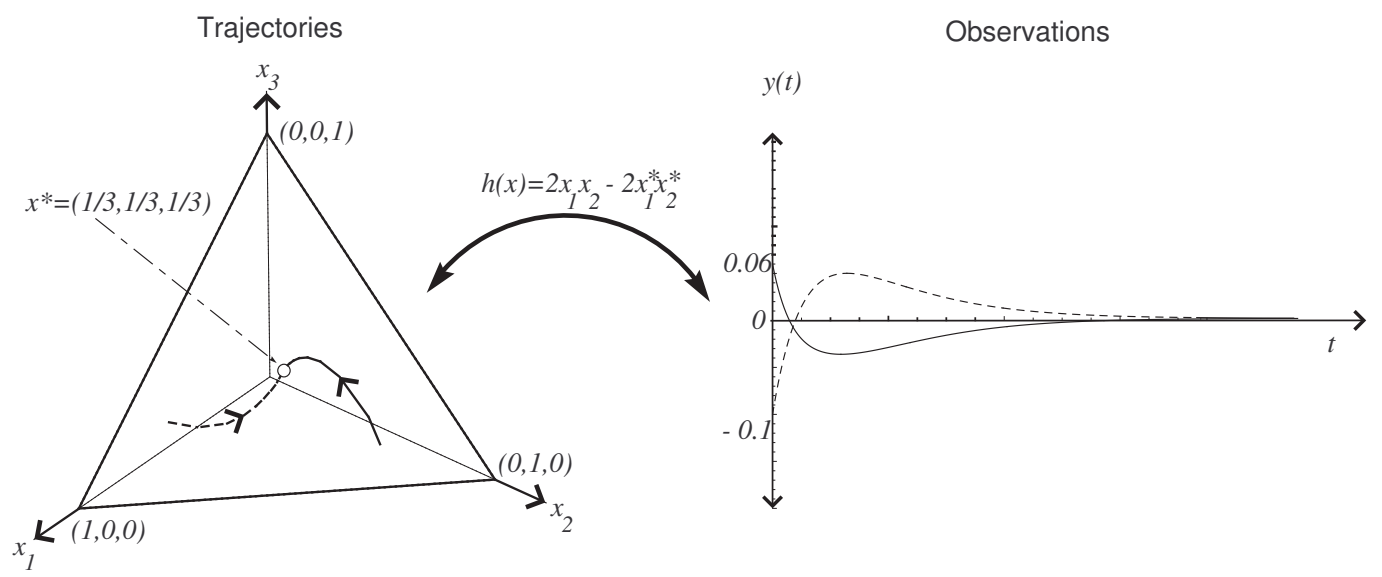

Fig. 1. Observability in the selection-mutation model

\section{Controllability into equilibrium by artificial selection}

As recalled in the introduction, the controllability problem of selection-mutation processes was studied in Scarelli and Varga (2002), where time-dependent mutation rates were considered as control functions. In this section the controllability of selection-mutation models is considered, where artificial selection is modelled in terms of time-dependent fitness parameters as control functions (selection strategies) of the form $w_{i j}+u_{i j}(t)$. 
Now we set up a general model of artificial selection. For each $i, j \in\{1, \ldots, n\}$, let $R_{i j}$ be an $n \times n$ matrix with all entries equal to zero except that with indices $i, j$, which is 1 , if genotypes $A_{i} A_{j}$ is artificially selected, and zero otherwise. In terms of mapping

$$
\psi: \mathbb{R}^{n \times n} \rightarrow \mathbb{R}^{n \times n}, \quad \psi(u):=\sum_{i, j=1}^{n} u_{i j} R_{i j},
$$

as a result of artificial selection we have a modified selection matrix $W+\psi(u)$.

With this notation, identifying $\mathbb{R}^{n \times n}$ with $\mathbb{R}^{n^{2}}$, for the dynamic model of artificial selection we have

$\dot{x}_{i}=x_{i}\left[(W x)_{i}-<x, \bar{w}(x)>\right]+x_{i}\left[(\psi(u) x)_{i}-<x, \psi(u) x>\right] \quad(i \in\{1, \ldots, n\})$.

Now we recall some concepts from nonlinear control theory.

Given $n, r \in \mathbb{N}$, let $F: \mathbb{R}^{n} \times \mathbb{R}^{r} \rightarrow \mathbb{R}^{n}$ be a continuously differentiable function. For a reference control value $u^{*} \in \mathbb{R}^{r}$, let $x^{*} \in \mathbb{R}^{n}$ be such that $F\left(x^{*}, u\right)=0$. For technical reason we shall need a rather general class of controls. Let us fix a time interval $[0, T]$, and for each $\epsilon>0$ define the class of controls

$$
\mathcal{U}_{\epsilon}[0, T]:=\left\{u \in L_{\infty}^{r}[0, T] \mid\|u(t)\|_{\infty}<\epsilon \text { for almost every } t \in[0, T]\right\} .
$$

From Lemma A.1 of the Appendix it follows that there exists $\epsilon_{0}>0$ such that for all $u \in \mathcal{U}_{\epsilon_{0}}[0, T]$ and $x^{0} \in \mathbb{R}^{n}$ with $\left\|x^{0}-x^{*}\right\|<\epsilon_{0}$ the control system

$$
\dot{x}(t)=F\left(x(t), u^{*}+u(t)\right) \quad(\text { for a.e. } t \in[0, T])
$$

has a unique solution with $x(0)=x^{0}$. We notice that $x^{*}$ is an equilibrium state for the zero-control system.

Definition 4.1. A regular $k$-dimensional submanifold $N \subset \mathbb{R}^{n}$ is said to be locally invariant for small controls with respect to system (21) at $x^{*}$, if there exists $\epsilon_{1} \in\left(0, \epsilon_{0}\right)$ such that $x(0) \in N,\left\|x(0)-x^{*}\right\|<\epsilon_{1}, u \in \mathcal{U}_{\epsilon_{1}}[0, T]$ imply that for solution $x$ of system (21) we have

$$
x(t) \in N \quad(t \in[0, T]) .
$$

Definition 4.2. Suppose that $N$ is as required in Definition 4.1. System (21) is called locally controllable into $x^{*}$ within $N$, if there exists $a \delta>0$ and a control $u \in \mathcal{U}_{\epsilon_{1}}[0, T]$ such that from any initial state $x(0) \in N$ with $\left\|x(0)-x^{*}\right\|<\delta$, for solution $x$ of (21) we have $x(T)=x^{*}$. 
For the controllability of a population into equilibrium we shall apply the following sufficient condition

Theorem 4.3. (Varga 1987) Assume that for the matrices

$$
L:=\frac{\partial F}{\partial x}\left(x^{*}, u^{*}\right) \quad, \quad B:=\frac{\partial F}{\partial u}\left(x^{*}, u^{*}\right)
$$

we have

$$
\operatorname{rank}\left(B|L B| \ldots \mid L^{n-1} B\right)=k .
$$

Then system (21) is locally controllable into $x^{*}$ within $N$.

For an illustration of the application of Theorem 4.3 to a controlled selectionmutation model, let us consider a three-allele system where homozygotes $A_{1} A_{1}$ and $A_{2} A_{2}$ as artificially selected. Now for the control model (20), in the definition of function $\psi$ we have the only nonzero matrices

$$
R_{11}:=\left[\begin{array}{ccc}
1 & 0 & 0 \\
0 & 0 & 0 \\
0 & 0 & 0
\end{array}\right] \quad, \quad R_{22}:=\left[\begin{array}{ccc}
0 & 0 & 0 \\
0 & 1 & 0 \\
0 & 0 & 0
\end{array}\right] .
$$

Let $x^{*} \in \stackrel{\circ}{\Delta}_{3}$ be a polymorphic equilibrium of the corresponding dynamics (20), $L$ and $B$ the matrices defined in Theorem 4.3. For $B$ we get

$$
B=\left[\begin{array}{cc}
\left(x_{1}^{*}\right)^{2}\left(1-x_{1}^{*}\right) & -x_{1}^{*}\left(x_{2}^{*}\right)^{2} \\
-\left(x_{1}^{*}\right)^{2} x_{2}^{*} & \left(x_{2}^{*}\right)^{2}\left(1-x_{2}^{*}\right) \\
-\left(x_{1}^{*}\right)^{2} x_{3}^{*} & -\left(x_{2}^{*}\right)^{2} x_{3}^{*}
\end{array}\right],
$$

since for any polymorphic equilibrium $x^{*}$, the determinant formed from the second and third rows of $B$ is nonzero. Furthermore,

$$
\operatorname{rank}\left[B|L B| L^{2} B\right]=3
$$

can not hold because the well-known sufficient condition for local controllability of Lee and Markus (1967) our system would be locally controllable into $x^{*}$ (in $\mathbb{R}^{3}$ ), in contradiction with the local invariance of $\stackrel{\circ}{\Delta}_{3}$ for small controls at $x^{*}$. Hence

$$
\operatorname{rank}\left[B|L B| L^{2} B\right]=2,
$$

and applying Theorem 4.3 we obtain the following 
Theorem 4.4. In the considered three-allele case, the population can be controlled into equilibrium from nearby states with small controls, applying a "soft" artificial selection strategy.

Now we numerically illustrate this theorem by the following

Example 4.5. Let us consider a three-allele model of the form (20), where all heterozygotes are equally fitter than the homozygote

$$
W:=\left(\begin{array}{ccc}
1 & 2 & 2 \\
2 & 1 & 2 \\
2 & 2 & 1
\end{array}\right) \quad, \quad M:=\left(\begin{array}{ccc}
-1 & 0 & 1 \\
1 & -2 & 1 \\
0 & 2 & -2
\end{array}\right) .
$$

From Theorem 2.1 we obtain that

$$
x^{*}=\frac{W^{-1} \mathbf{1}}{<W^{-1} \mathbf{1}, \mathbf{1}>}=\left(\begin{array}{c}
1 / 3 \\
1 / 3 \\
1 / 3
\end{array}\right) \in \operatorname{ker} M
$$

is a polymorphic equilibrium for the corresponding system (20). Now supposing that genotypes $A_{1} A_{1}$ and $A_{2} A_{2}$ are controlled, from Theorem 4.4 we get that the population can be controlled into the equilibrium in given time, at least from nearby states, applying a "soft" artificial selection on a given time interval.

\section{Discussion}

We have considered a continuous-time selection-mutation process from three aspects which may be important for monitoring and conservation of the genetic state of a population. The first issue was the existence and calculation of a polymorphic equilibrium.

As for the monitoring, we supposed that instead of the genetic state (allele frequencies) certain phenotype frequencies are at our disposal. The observability concept and the corresponding sufficient condition of nonlinear mathematical 
systems theory provide a possibility to recover the underlying genetic process from phenotypic observation.

In order to reestablish an equilibrium of the genetic composition, the concept of controllability can be applied. For a nonlinear control model of artificial selection, considering a concrete selection situation it is shown that, even in the presence of mutation, the population can be controlled into equilibrium in given time, applying small intervention into the selection process.

Our considerations can be extended in order to build a selection strategy in accordance with the structure of the genotype-phenotype correspondence. Indeed, this can be realized when genotypes $A_{i} A_{j}$ and $A_{k} A_{l}$ are selected in the same way, if they have the same phenotype.

Finally we notice that since the technique of nonlinear systems theory applied to controllability problems makes it possible to deal only with autonomous systems, it can be applied only in the case of a stationary environment. At the same time, however, as already noticed in Varga et al. (2003), the monitoring problem can also be treated in case of a changing environment, when certain abiotic effects are modeled as time-dependent parameters formally considered as control functions.

\section{Acknowledgements}

This publication has been realized in the framework of the Hungarian-Spanish intergovernmental scientific and technological collaboration, with the support of the Scientific and Technological Innovation Fund (of Hungary) and the Ministry of Education and Sciences (of Spain), and has also been supported by the Hungarian National Scientific Research Fund (OTKA T037271). Finally, the authors are grateful to the anonymous referees for their valuable comments. 


\section{References}

Akin, E., 1979. The Geometry of Population Genetics. Lecture Notes on Biomathematics. Springer, Heidelberg, Berlin, New York.

Bürger, R. Mathematical properties of mutation-selection models. Genetica 102/103, (1998), 279-298.

Crow, J.F.; Kimura, M. An Introduction to Population Genetics Theory. Harper and Row, New York, (1970).

Farkas, G. Local controllability of reaction. J. Math. Chem, 24, (1998a), 1-14.

Farkas, G. On local observability of chemical systems. J. Math. Chem, 24, (1998b), 15-22.

Fisher, R.A. The Genetical Theory of Natural Selection. Clarendon Press, Oxford, (1930).

Fisher, R.A. The Genetical Theory of Natural Selection. Dover Publications, New York, (1958).

Fogel, David B. Evolutionary Computation: Towards a New Philosophy of Machine Intelligence (second edition). IEEE Press, (2000).

Gámez, M.; Carreño, R.; Kósa, A.; Varga, Z., 2003. Observability in strategic models of viability selection. BioSystems 71 (3), 249-255.

Garay, J.; Garay, B. When does a sexual population realize all phenotypic states? J. Math. Biol., 37, (1998), 146-154.

Hadeler, K.P. Stable polymorphisms in a selection model with mutation. SIAM J. Appl. Math. 36, (1981), 1-7.

Hofbauer, J. The selection mutation equation. J. Math. Biol., 23, (1985), 41-53.

Hofbauer, J.; Sigmund, K., 1988. The Theory of Evolution and Dynamical Systems, 1988. Cambridge: Cambridge University Press.

Hofbauer, J.; Sigmund, K., 1998. Evolutionary Games and Population Dy- 
namics. Cambridge University Press, Cambridge.

Kalman, R.E.; Falb, P.L.; Arbib, M.A., 1969. Topics in Mathematical System Theory. McGraw-Hill, New York.

Kósa, A.; Varga, Z., 1996. Controllability of evolutionary game dynamics. Bull. Univ. Agric. Sci. Gödöllo (II), 75, 43-49.

Lee, E.B.; Markus, L., 1967. Foundations of Optimal Control Theory. Wiley, New York.

López, I.; Gámez, M.; Carreño, R.; Varga, Z. Recovering genetic processes from phenotypic observation. Proceedings 5th. European Conference on Mathematical and Theoretical Biology 2002. Milán, 2-6 julio 2002, 356-361.

López, I.; Gámez, M.; Carreño, R.; Varga, Z. Optimization of mean fitness of a population via artificial selection. Proceedings volume from the 12th IFAC Workshop. Viségrad (Hungría), 30 junio - 2 julio 2003, 147-150.

Moran, P.A.P. Global stability of genetical systems governed by selection and mutation. Math. Proc. Camb. Phil. Soc., 80, (1976), 331-336.

Moran, P.A.P. Global stability of genetical systems governed by selection and mutation. II. Math. Proc. Camb. Phil. Soc., 81, (1977), 435-441.

Riesz, F.; Sz-Nagy, B. Functional analysis. Dover, (1992).

Scarelli, A.; Varga, Z., 2002. Controllability of selection-mutation systems. BioSystems 65 (2), 113-121.

Varga, Z., 1989. On controllability of Fisher's model of selection. In: Dafermos, C.M., Ladas, G., Papanicolaou, g. Proc. EQUADIFF Conference, 717-723.

Varga, Z., 1992. On observability of Fisher's model of selection. Pure Math. and Appl. Ser. B, 1, 15-25.

Varga, Z.; Zubiri, J.A. Equilibrium and stability of a Fisher type selectionmutation model. Abstr. Bot., 17, (1993), 341-344.

Varga, Z.; Scarelli, A.; Shamandy, A., 2003. State monitoring of a population system in changing environment. Community Ecology, 73-78. 
Zadeh, L.A.; Desoer, C.A. Linear System Theory. McGraw-Hill, New York, (1963).

\section{Appendix:}

First we recall a lemma on the continuous dependence of the solution on the control (Lee and Markus (1967), section 4.1).

Lemma A1. Let $\phi \in C^{1}\left(\mathbb{R}^{n} \times \mathbb{R}^{r}, \mathbb{R}^{n}\right), n, r \in \mathbb{N}$, let $\Omega \subset \mathbb{R}^{r}$ be a compact set and fix a $T>0$. For any measurable function (control) $u:[0, T] \rightarrow \Omega$ consider the system

$$
\dot{x}(t)=\phi(x(t), u(t)) \quad \text { for almost all } t \in[0, T] .
$$

(a) Suppose that for a measurable control $\bar{u}:[0, T] \rightarrow \Omega$, a solution $\bar{x}$ of (22) is defined on the interval $[0, T]$. Then there exists an $\epsilon_{0}>0$ with the following property: for all $\epsilon \in\left(0, \epsilon_{0}\right)$ and measurable control $u_{\epsilon}:[0, T] \rightarrow$ $\Omega$ satisfying $\left\|u_{\epsilon}(t)-\bar{u}(t)\right\|<\epsilon$ except a set of measure $\epsilon$, we have that any solution $x_{\epsilon}$ corresponding of system (22) with $\left\|x_{\epsilon}(0)-\bar{x}(0)\right\|<\epsilon$ is defined on $[0, T]$, moreover $\left\|x_{\epsilon}(t)-\bar{x}(t)\right\|<\epsilon \quad(t \in[0, T])$ holds.

(b) With the conditions and notation of $(a)$ we have that $x_{\epsilon} \rightarrow \bar{x}$ uniformly on $[0, T]$ when $\epsilon \rightarrow 0$.

Using this lemma we shall prove the following

Lemma A2. $\stackrel{\circ}{\Delta}_{n}$ is locally invariant for small controls with respect to system (20), at any equilibrium $x^{*} \in \stackrel{\circ}{\Delta}_{n}$.

Proof. Apply part (a) of Lemma A1 with $r:=n^{2}, \Omega:=[-1,1]^{n^{2}}, \bar{u}(t):=0$ and $\bar{x}(t):=x^{*}(t \in[0, T])$, using the notation $\mathcal{U}_{\epsilon}[0, T]$ introduced in section 4 .

Then there exists an $\epsilon_{0} \in(0,1)$ such that for all $\epsilon \in\left(0, \epsilon_{0}\right), u \in \mathcal{U}_{\epsilon}[0, T]$ and $z \in \stackrel{\circ}{\Delta}_{n}$ with $\left\|z-x^{*}\right\|<\epsilon$, the solution $x_{u}$ of system (20) with initial value $z$ is defined on $[0, T]$, moreover $\left\|x_{u}(t)-x^{*}\right\|<\epsilon$. It is clear that by the choice of $\epsilon_{0}$ we can also guarantee that the coordinates of $x_{u}(t)$ are positive $(t \in[0, T])$.

Fix $\epsilon \in\left(0, \epsilon_{0}\right)$, a control $u \in \mathcal{U}_{\epsilon}[0, T]$ and $z \in \stackrel{\circ}{\Delta}_{n}$ with $\left\|z-x^{*}\right\|<\epsilon$. Since $u$ is measurable, in $\mathcal{U}_{1}[0, T]$ there exist a sequence $\left(u_{k}\right)$ of step functions (piece-wise constant functions) and a set $E_{0} \subset[0, T]$ of measure zero such that $\lim \left(u_{k}(t)\right)=u(t)\left(t \in[0, T] \backslash E_{0}\right)$, see for example Riesz and Sz-Nagy (1992). Applying the well-known invariance of the simplex for the dynamics 
without control (see eg. Hofbauer Sigmund, 1998) by "pieces", always with the same initial value $z$, we obtain that for the corresponding solutions $x_{u}$ we have $x_{u}(t) \in \Delta_{n}(t \in[0, T])$. In order to apply the classical theorem of Egorov (see Riesz and Sz-Nagy (1992)) define $E:=[0, T] \backslash E_{0}$. Then $\mu(E)=T$ and for all $l \in \mathbb{N}$ there exists a subset $E_{l} \subset E$ with $\mu\left(E_{l}\right)>T-\frac{1}{l}$ such that $\left(u_{k}\right)$ tends to $u$ uniformly in $E_{l}$. Therefore, for all $l \in \mathbb{N}$ there exists a $k_{l} \in \mathbb{N}$ such that $\left\|u_{k_{l}}(t)-u(t)\right\|<\frac{1}{l} \quad(t \in E)$. By part $(b)$ of Lemma A1, the sequence of solutions $\left(x_{u_{k_{l}}}\right)$ tends to $x_{u}$ uniformly in $[0, T]$. Since $x_{u_{k_{l}}} \in \Delta_{n} \quad(t \in[0, T])$ and $\Delta_{n}$ is closed, we obtain that

$$
x_{u}(t) \in \Delta_{n} \quad(t \in[0, T]),
$$

which with the positivity of $x_{u}(t)$ proved before, completes the proof.

Note. In particular, taking $u \equiv 0$ we obtain that $\stackrel{\circ}{\Delta}_{n}$ is locally positively invariant for the dynamics of selection-mutation (2), which implies the invariance of simplex for this dynamics. 